CLINICAL PRACTICE

Clinical Vignettes

\title{
Syphilitic Proctitis Presenting as a Rectal Mass: a Case Report and Review of the Literature
}

\author{
Jessica K. Costales-Cantrell, DO, MBS ${ }^{\top}$ (D), Elizabeth Y. Dong, MD², Bechien U. Wu, MD², \\ and Jim H. Nomura, MD ${ }^{3}$ \\ 'Division of Infectious Diseases, University of California Invine Medical Center, 101 City Drive South, City Tower Suite 400, Orange, CA, USA; \\ ${ }^{2}$ Department of Gastroenterology, Kaiser Permanente Los Angeles Medical Center, Los Angeles, CA, USA; ${ }^{3}$ Division of Infectious Diseases, Kaiser \\ Permanente Los Angeles Medical Center, Los Angeles, CA, USA.
}

J Gen Intern Med 36(4):1098-101

DOI: $10.1007 / \mathrm{s} 11606-020-06414-9$

(C) Society of General Internal Medicine 2021

\section{CASE PRESENTATION}

A 32-year-old previously healthy man initially presented to the emergency department with lower abdominal pain, proctalgia, and diarrhea with hematochezia for 2 weeks. Computed tomography (CT) with intravenous contrast demonstrated wall thickening of the distal sigmoid colon and rectum with perirectal, pelvic, and retroperitoneal lymphadenopathy (Fig. 1a, b). He was initially diagnosed with infectious colitis and discharged from the emergency department with a course of ciprofloxacin and metronidazole.

Due to worsening symptoms and inability to tolerate oral intake, the patient returned to the emergency department 3 days later. Further investigation revealed a history of anoreceptive intercourse with men without barrier protection. The patient denied a history of prior sexually transmitted infections.

Physical exam was notable for temperatures up to $101.8^{\circ}$ Fahrenheit, lower abdominal tenderness to palpation, and painful inguinal lymphadenopathy. Rectal exam was notable for scant bright red blood with palpation of a rectal mass. Neurological exam was normal. Complete blood count revealed leukocytosis of 13,000 cells $/ \mathrm{mm}^{3}$. Repeat CT demonstrated irregular thickening of the rectum with extensive perirectal lymphadenopathy, up to $1.5 \mathrm{~cm}$ in size, suspicious for malignancy. Flexible sigmoidoscopy revealed nonbleeding ulcers with friable, raised ulcerated mucosa in the rectum (Fig. 2). Rectal biopsies revealed focal ulceration, noncaseating granulomas with multinucleated giant cells without histological evidence of malignancy. Warthin-Starry stain and immunohistochemical staining (IHC) were negative for spirochetes and fungal elements (Fig. 3).

Serologic testing for human immunodeficiency virus by 4 th generation assay and herpes simplex virus 1 and 2 were

This case was presented at the American College of Physicians Southern California Regional Abstract Competition, October 2018.

Received May 15, 2020

Accepted December 8, 2020

Published online January 19, 2021 negative. Nucleic acid amplification testing for Chlamydia trachomatis and Neisseria gonorrhoeae of the rectum were negative. Rapid plasma reagin (RPR) titer was 1:128, with a reactive Treponema pallidum antibody (Architect Syphilis TP Assay, Abbott) and Treponema pallidum particle agglutination (Serodia TP-TA Assay, Fujirebio). The diagnosis of syphilitic proctitis was made. Based on the high RPR titer and systemic symptoms, secondary stage of syphilis was suspected. As such, the patient was administered one dose of 2.4 million units of benzathine penicillin $\mathrm{G}$ with improvement of leukocytosis and fevers. No Jarisch-Herxheimer reactions were noted. The patient was seen on follow-up 1-week post hospital discharge with resolved abdominal pain, diarrhea, and hematochezia with no abdominal tenderness on exam. The resolution of symptoms after antibiotic therapy confirmed the diagnosis of syphilitic proctitis.

\section{DISCUSSION}

\section{Clinical Manifestations, Diagnosis, and Treatment of Syphilis}

Syphilis is caused by the spirochete Treponema pallidum. The disease is transmitted both sexually and vertically, but most commonly transmitted by sexual contact. ${ }^{1}$ The clinical presentation of the disease is divided into various stages: primary, secondary, latent, and tertiary syphilis. If left untreated, patients sequentially progress through the different stages of infection. Primary syphilis presents approximately 3 weeks after infection as a single painless chancre or multiple lesions at the site of inoculation with regional lymphadenopathy. The incubation period can last up to 90 days. ${ }^{1}$ Primary and secondary stages may overlap; however, secondary manifestations typically follow 6-8 weeks after primary lesions resolve. ${ }^{2}$ Clinical manifestations of secondary syphilis include systemic manifestations, such as a polymorphic rash, fever, and lymphadenopathy. This stage can also manifest as mucus lesions (presenting as a mucus patch involving the tongue, buccal mucosa, and lips) or genital mucus lesions (presenting as papules, ulcerations, and condyloma lata). The incubation period of secondary syphilis can last up to 6 months. ${ }^{1}$ After resolution of symptoms, patients enter the asymptomatic stage 


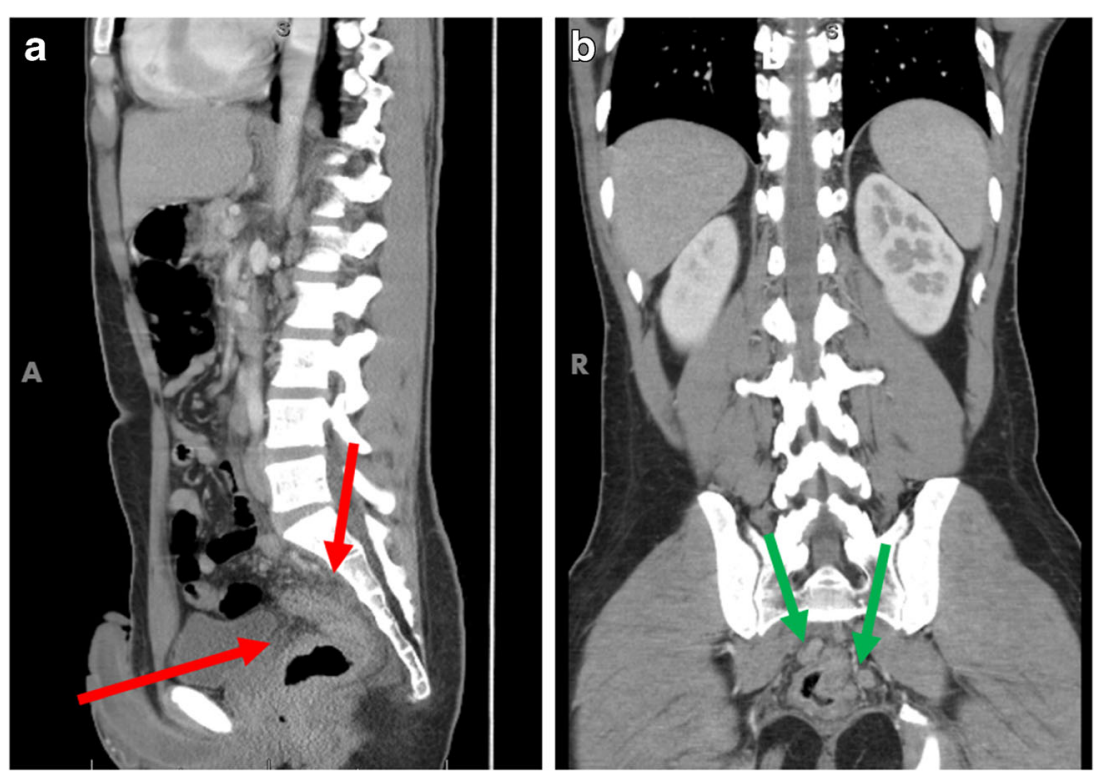

Fig. 1 CT scan of the abdomen and pelvis with intravenous contrast. Panel a is a sagittal view demonstrating abnormal wall thickening and inflammatory changes of the distal sigmoid colon and rectum (red arrows). Panel $b$ is a coronal view illustrating multiple enlarged perirectal lymph nodes (green arrows)

of latent syphilis. This particular stage is divided into early latent (occurring within 1 year of infection) and late latent (occurring after 1 year of infection). If left untreated, the disease progresses into tertiary syphilis, which can affect the cardiovascular and neurologic systems, or cause skin or visceral gummas. This stage is now considered rare, owing to widespread antibiotic use. ${ }^{1}$ Neurosyphilis is a condition that can manifest with ocular or otic symptoms, chronic meningitis, tabes dorsalis, or general paresis and can occur at any stage of syphilis. ${ }^{1,3}$

Diagnosis of syphilis is based on clinical findings and serologic testing. Most common serologic tests to diagnose primary, secondary, latent, and tertiary syphilis include a combination of nontreponemal tests (e.g., RPR titers) and treponemal testing, which include detection of antibodies against $T$. pallidum using enzyme immunoassay (EIA), microhemagglutination (MHA-TP), particle agglutination assays (TPPA), or fluorescent treponemal antibody absorption assay (FTA-ABS). ${ }^{3}$ Diagnosis of neurosyphilis requires a lumbar puncture for cerebrospinal (CSF) fluid analysis. The Venereal Disease Research Laboratory (VDRL) assay is considered the gold standard for diagnosis of neurosyphilis. ${ }^{1,3}$

Penicillin is the first-line treatment of syphilis as it is effective for all stages of syphilitic infection. ${ }^{2}$ Due to a slower rate of bacterial replication of T. pallidum, levels of antibiotic must be above the minimum inhibitory concentration (MIC) for at least $7-10$ days. ${ }^{2,3}$ For early syphilis (i.e., primary, secondary, and early latent syphilis), this can be achieved with one intramuscular injection of long-acting benzathine penicillin G. This is advantageous over shorter-acting antibiotic therapy due to improved adherence. Treatment of patients with late syphilis (i.e., late latent and tertiary) includes intramuscular benzathine penicillin $\mathrm{G}$ weekly for 3 weeks.
Treatment of neurosyphilis includes high-dose intravenous aqueous crystalline for 10-14 days, since the dose of intramuscular benzathine penicillin $G$ does not produce adequate levels in the CSF. Patients with a documented penicillin allergy are recommended to undergo desensitization and subsequent treatment with penicillin, as there is limited data for alternative antibiotic regimens which should be reserved for cases where treatment with penicillin is contraindicated. ${ }^{2}$

The goal of treatment is to achieve a clinical and serologic cure. Serologic cure of syphilis is defined as a decline of nontreponemal titers by a factor of 4 or more 6-12 months after treatment for early syphilis and 12-24 months for late syphilis. ${ }^{2}$ Patients who do not achieve the expected decline in nontreponemal titers are considered to have a serologic nonresponse and a lumbar puncture for CSF analysis is often recommended to evaluate for neurosyphilis. ${ }^{2}$

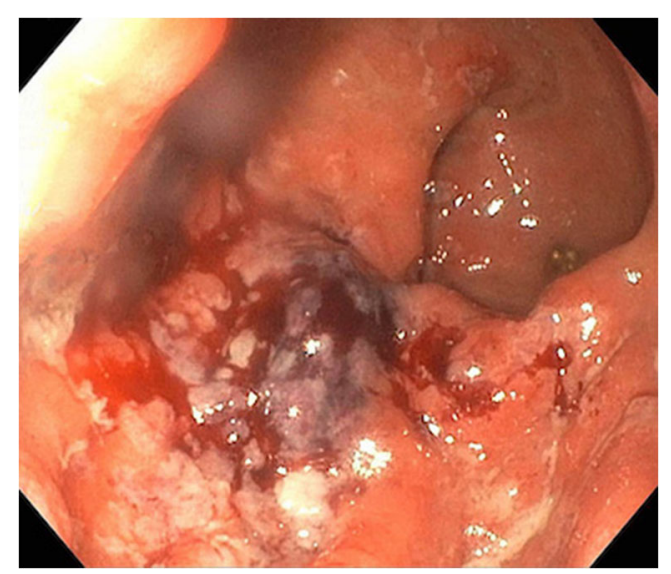

Fig. 2 Endoscopic view of rectum showing diffuse friable, ulcerated lesions, and gross inflammatory changes without a discrete mass 


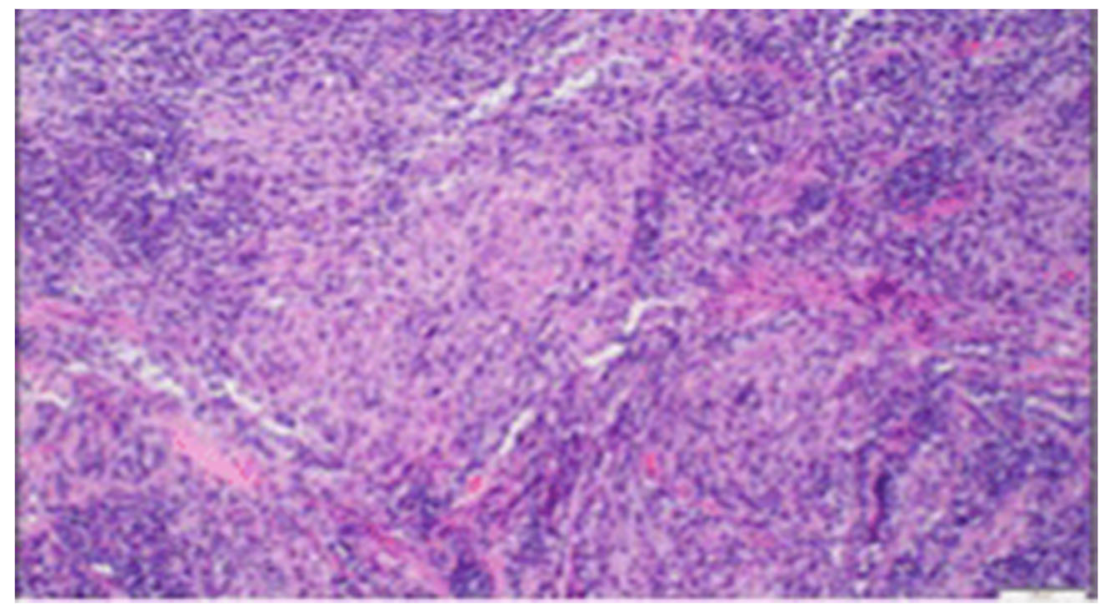

Fig. 3 Histological examination of the colonic mucosa showing focal ulceration and non-caseating granulomatous inflammation including scattered multinucleated giant cells on hematoxylin and eosin stain. No histological findings were suggestive of malignancy

\section{Epidemiology of Syphilis}

There has been a striking increase in the reported cases of syphilis in the United States (US) and other high- and middleincome countries. Particularly in the United States, the rate of primary and secondary $(\mathrm{P} \& \mathrm{~S})$ syphilis cases has been steadily increasing since the turn of the century. In 2018, there were 115,000 syphilis cases, 35,000 of which were P\&S syphilis, representing the highest number reported since $1991 .{ }^{4}$ This poses a public health concern given this is the most infectious stage of syphilis. The 2018 STD Surveillance report from the Centers of Disease Control (CDC) reported a $71 \%$ increase in $\mathrm{P} \& \mathrm{~S}$ syphilis and a $185 \%$ increase rise in congenital syphilis since 2014, the latter owing to a doubled rate of P\&S syphilis among childbearing women. ${ }^{4}$ The men who have sex with men (MSM) population accounted for the majority of $\mathrm{P} \& \mathrm{~S}$ syphilis cases in the US in both 2016 and 2018, accounting for $58 \%$ and $53.5 \%$ of cases, respectively. ${ }^{4,5}$ Forty-two percent of this population were co-infected with HIV, indicating a strong association between syphilis and HIV infection. ${ }^{2}$ Reasons for this have been attributed to the tendency for more sexual partners, engagement in oral and anoreceptive intercourse which enables efficient transmission of the organism, decreased condom use, and complex socioeconomic and behavioral factors that prevent access to health services. ${ }^{5}$

\section{Syphilitic Proctitis}

Syphilitic proctitis is a rare manifestation of syphilis infection that can manifest as chancres, mass-like lesions, and ulcers in the anal canal. ${ }^{6}$ There have been several case studies of syphilitic proctitis, often reported in the MSM population and/or patients with human immunodeficiency virus (HIV). ${ }^{6-11}$

Common symptoms include lower abdominal pain, tenesmus, weight loss, hematochezia, and rectal pain. Rarely, it can be asymptomatic. ${ }^{12}$ Imaging may reveal mass-like lesions with surrounding lymphadenopathy and inflammatory changes that are easily mistaken for malignancy, non-specific proctitis, inflammatory bowel disease, or other infectious causes of proctitis. ${ }^{9,13,14}$ Physical exam may show a rectal mass or anal fissure. ${ }^{15}$ CT scan usually shows rectal wall thickening with pelvic lymphadenopathy. ${ }^{13,16}$

Endoscopy shows diffuse edema, friable mucosa, multiple erosions, and ulcerated irregular rectal masses that can range from 15 to $40 \mathrm{~mm}$ in size..$^{9-11,17,18}$ Histologically, biopsies of the rectum show mild acute or chronic non-specific inflammation with lymphohistiocytic infiltration with rare noncaseating granulomas. ${ }^{9-11,17,19}$ Silver stains, such as Steiner or Warthin-Starry stains, may show spirochetes, though both stains have low sensitivity and specificity. IHC staining has improved diagnostic accuracy over Warthin-Starry stains, but it still does not have sufficient sensitivity for ruling out syphilis. Studies comparing standard silver stains to IHC staining in skin biopsies from patients with secondary syphilis noted IHC staining was a more sensitive method for detection than silver staining, with sensitivities ranging from 71 to $90 \%$ versus $41-$ $60 \%$, respectively. ${ }^{20,21}$ However, a report of sensitivity as low as $10 \%$ has been reported in one study with IHC staining. ${ }^{11}$ When organisms are not detected on biopsy, it can be explained by sampling error, variable distribution of organisms, or previous treatment. ${ }^{11,19}$

Treatment of syphilitic proctitis includes benzathine penicillin $\mathrm{G}$. There is considerable variability among the published case reports regarding the dosing and frequency of penicillin used for syphilitic proctitis, ranging from a single dose to once weekly dosing for 3 weeks. After treatment, there is often a rapid improvement in clinical symptoms. Follow-up endoscopy is often obtained, revealing healing ulcers or complete resolution of abnormalities and rectal masses. ${ }^{6,7,9}$

It is important to note that the differential diagnosis of proctitis in the MSM population is usually due to infectious causes. In the right host, noninfectious causes, including inflammatory bowel disease, radiation proctitis, and ischemic colitis, should also be considered. Infectious causes of proctitis with concomitant extensive colitis are commonly caused by Escherichia coli, Shigella, Campylobacter, and Clostridioides difficile. ${ }^{22}$ On the other hand, isolated infectious proctitis is 
often caused by organisms that are sexually transmitted, most commonly Neisseria gonorrhoeae (30\%) and Chlamydia trachomatis (19\%), followed by herpes simplex virus 2 $(16 \%)$ and syphilis $(2 \%){ }^{22,23}$ Other rare causes of infectious proctitis include amebiasis and Mycoplasma genitalium. ${ }^{24}$ Coinfection rates have been reported as high as $10 \%$, usually found in conjunction with $C$. trachomatis lymphogranuloma venereum serotype. ${ }^{11,15,22-25}$

Due to the non-specific findings of syphilitic proctitis and rarity of the condition, diagnosis is challenging, often placing patients at risk of being misdiagnosed and treated inappropriately. ${ }^{6,17,19,26-28}$ As such, a high index of suspicion is needed to arrive at an accurate diagnosis, which can be aided with obtaining a thorough history, including a detailed sexual history. Due to the difficulty of visualizing a causative organism on biopsy, diagnosis is achieved by a combination of clinical presentation, serologies, and endoscopy. ${ }^{6,11}$ This was particularly evident in this case, where a detailed clinical and sexual history was the key in raising the clinical suspicion for syphilis, leading to serologic testing. Based on the patient's prompt and complete resolution of symptoms after treatment, the diagnosis of syphilitic proctitis was able to be confirmed.

\section{CONCLUSION}

This case emphasizes that the diagnosis of syphilitic proctitis should be included in the differential diagnosis of patients presenting with rectal pain and a history of anoreceptive intercourse, especially in the MSM or HIV positive population. Since cases of syphilis is increasing in the United States and globally, awareness of this atypical presentation of the condition is needed to allow for appropriate diagnosis, treatment, and prevent late complications of undiagnosed syphilis.

Acknowledgments: We would like to thank Raffi Ourfalian, DO, for assistance in selecting radiographic images for the article and Miguel Fernando Palma Diaz, MD, for aid in obtaining histology images.

Corresponding Author: Jessica K. Costales-Cantrell, DO, MBS; Division of Infectious Diseases, University of California Irvine Medical Center, 101 City Drive South, City Tower Suite 400, Orange, CA 92868, USA (e-mail: Jess.costales@gmail.com).

\section{Compliance with Ethical Standards:}

Conflict of Interest: The authors declare that they do not have a conflict of interest.

\section{REFERENCES}

1. Singh AW, Romanowski. Syphilis: review with emphasis on clinical, epidemiologic and some biologic features. Clin Microbiol Rev 1999;12(2)187-209.

2. Ghanem KG, Ram SR, Rice PA. The Modern Epidemic of Syphilis. N Engl J Med. 2020; 382:845-54.

3. Peeling RW, Mabey D, Kamb ML, Chen X, Radolf JD, Benzaken AS Syphilis. Nature Reviews Disease Primers. 2017;3:17073.
4. Centers for Disease Control and Prevention. Sexually Transmitted Disease Surveillance 2018, U.S. Department of Health and Human Services, accessed 20 August 2020, https://www.cdc.giv/std/stats18/ default.htm

5. Mayer KH. Old pathogen, new challenges: a narrative review of the multilevel drivers of syphilis increasing in American men who have sex with men. Sex Transm Dis. 2018;45(9S suppl):S38-S41.

6. Yang JF, Peng L, Siddiqui AA, Mayorga CA. Syphilitic proctitis. Proc Mayl Univ Med Cent. 2016;29(3):327-328.

7. Nazemi MM, Mushner DM, Schell RF, Milo S. Syphilitis Proctitis in a Homosexual. 1975;231(4):389.

8. Akamdar K, Martin RJ, Ichinose $\mathbf{H}$. Syphilitic proctitis. The american journal of digestive diseases. 1977;22(8):701-704.

9. Teitelman M, Wilson JA, Guy CD. Image of the month. Syphilitic Proctitis. Clin Gastroenterol Hepatol. 2008;6(4):xxvi.

10. Adachi E, Koibuchi T, Okame $\mathbf{M}$ et al. Case of secondary syphilis presenting with unusual complications: syphilitic proctitis, gastritis, and hepatitis. J Clin Microbiol. 2011;49(12):4934-46.

11. Arnold CA, Limketkai BN, Illei PB, Montgomery E, Voltaggio L. Syphilitic and lymphogranuloma venereum (LGV) proctocolitis: clues to a frequently missed diagnosis. Am J Surg Pathol. 2013;37(1):38-46.

12. Alan-Biltz LT, Beaird OE, Dry SM, Kaneshiro, M, Klausner, JD. A Case of Asymptomatic Syphilitic Proctitis. Sex Transm Dis. 2019;46(6):e68e69.

13. Bronson T, Behdad A, Pezhouh MK. Syphilitic proctitis with clinical features of lymphoma. Clin Gastroenterol Hepatol. 2020; S15423565(20)30201-9.

14. Gomez, NA, Leon, CJ, Rojas JE, Arevalo, CA. Syphilitic rectal ulcer associated with perforation of the hard palate: case report. Acta Gastroenterol Latinoam. 1997;27(4):263-65.

15. Siddiqui WT, Schwartx HM. Infectious Proctitis. N Engl J Med. 2020;382(17): 1638.

16. Chiang $\mathbf{T}$, Hsieh KL, Kung $\mathbf{C H}$. Syphilitic proctitis imitating rectal malignancy. Eur J Gastroenterol Hepatol. 2020;32(2):205-86.

17. Zhao W, Liu J, Li Y. Syphilitic proctitis mimicking rectal cancer: a case report. World J Gastrointest Pathophysiol. 2010;1(3):112-114.

18. Teng KT, Lee JY, Chang $\mathbf{C C}$. An easily overlooked cause of mucus and bloody material passage during defecation in a 47-year old man. Gastroenterology. 2018;154(8):e3-e4.

19. Gopal P, Shah RB. Primary Anal Canal Syphilis in Men: The Clinicopathologic Spectrum of an Easily Overlooked Diagnosis. 2015;139(9):1156-60.

20. Phelps RG, Knispel J, Tu ES, Cernainu G, Saruk M. Immunoperoxidase technique for detecting spirochetes in tissue sections: comparison with other methods. Int J Dermatol. 2000;39:609-13.

21. Hoang MP, High WA. Molberg KH. Secondary syphilis: a histologic and immunohistochemical evaluation. J Cutan Pathol. 2004;31:595-99.

22. Hoentjen F, Rubin DT. Infectious proctitis: when to suspect it is not inflammatory bowel disease. Dig Dis Sci. 2012;57(2):269-73.

23. Klausner JD, Kohn R, Kent C. Etiology of clinical proctitis among men who have sex with men. Clin Infec Dis. 2004;38(2):300-302.

24. Bissessor M, Tabrizi, SN, Bradshaw, CS, et al. The contribution of mycoplasma genitalium to the aetiology of dexually acquired infectious proctitis in men who have sex with men. Clin Microbiol Infect. 2016;22(3):260-65.

25. Sousa M, Pinho R, Rodrugues A. Infectious proctitis due to syphilis and chlamydia: an exuberant presentation. Rev Esp Enferm Dig. 2019;111(10):813-819.

26. Samenius B. Primary syphilis for the anorectal region. Proc R Soc Med 1966;59(7): 629-631.

27. Guinn TC, Corey L, Chaffee RG, Schuffler MD, Bracanto FP, Holmes KK. The etiology of anorectal infections in homosexual men. Am J Med. 1981;7(13):395-406.

28. Song SH, Jang I, Kim BS, Kim ET, Woo S, Park MJ, et al. A case of primary syphilis in the rectum. J Korean Med Sci. 2005;20:886-887.

Publisher's Note: Springer Nature remains neutral with regard to jurisdictional claims in published maps and institutional affiliations. 